\title{
Fingerprint Indexing Based on LAS Registration
}

\author{
Tong Liu' ${ }^{1}$ Chao Zhang ${ }^{l}$ and Pengwei Hao ${ }^{1,2}$ \\ ${ }^{1}$ National Laboratory on Machine Perception, Peking University, Beijing, 100871, China \\ ${ }^{2}$ Department of Computer Science, Queen Mary, University of London, E1 4NS,UK \\ Email: chzhang@cis.pku.edu.cn
}

\begin{abstract}
Fingerprint indexing is an efficient technique that greatly improves the performance of fingerprint based person authentication systems by reducing the number of comparison. In this paper, we propose an indexing method based on fingerprint registration with a novel feature called local axial symmetry (LAS). The location and direction estimation of reference point are achieved in a straightforward way after the LAS field is achieved. Then the registered orientation field is utilized as a feature vector to perform the following indexing. A new scheme of the experiment is introduced and satisfactory experimental results are achieved on FVC2000 DB2 that the average search space is only $2.34 \%$ of all fingers in the condition of equal-sized training set and testing set.
\end{abstract}

Index Terms-Fingerprint, Local Axial Symmetry, Indexing

\section{INTRODUCTION}

Fingerprints have long been used as a reliable biometric feature for person identification. However, fingerprint matching is computationally demanding especially for a large database. Fingerprint indexing algorithms reduce the number of comparison by selecting most probable candidates and sort them by the similarity to the input one [1]. For indexing technique performs better than exclusive classification considering the size of space that need to be searched [2], many indexing algorithms have been proposed recently.

In [1] and [3], the triplets of minutiae are used in the indexing procedure. These methods focus on the detailed information of fingerprints and ignore the macro information which is more robust to local noise. A.K. Jain et al [4] use the features around a core point of a Gabor filtered image to realize indexing. An indexing algorithm was proposed based on correlation of the robustly detected singular points in [5]. Efforts on combining methods, such as [6] and [7] can also be seen in the literature.

As the most important feature in fingerprint image, the position and direction of reference point can lead a dependable registration to dependably regularize the different fingerprint images. Further the registered orientation field contains abundant information to describe the similarity of fingerprints. We develop an efficient indexing algorithm according to these facts in this paper and obtain satisfactory experimental results.

This paper is organized as follows. In Section 2, the LAS is introduced and its property is analyzed. In Section 3, position and direction of reference point are extracted based on LAS field and the registered orientation field is obtained to realize fingerprint indexing. In Section 4, some experimental results are presented. Finally, the conclusions are drawn in Section 5.

\section{LOCAL AXIAL SYMMETRY}

In fingerprint analysis field, orientation field (OF) and Poincaré index (PI) are two important features. For convenience, we name the pixel-wise computed OF as POF and the block-wise computed OF as BOF. PI which was first introduced in [8], is a widely exploited feature in detection of singular points. In our view, reference point detection algorithm based on PI is noise-sensitive and incapacity to the arch type fingerprints, so we propose a novel feature also derived from OF but more consistent and compatible to detect reference points.

As can be easily seen, the $\mathrm{OF}$ in most regions of fingerprint images shows good axial symmetry such as the first two images in Fig 1. But in region like that in the last image, even axis which maximizes the axial symmetry value of the regions is picked out, the divided two parts seem to be very dissimilar to each other. Due to its rareness, if the latter type of region can be stably recognized, it can be utilized to detect the reference points of fingerprint images. Our LAS is proposed to realize this motivation.

To measure the LAS of a given region, we first cut out a circle in POF, which is the doubled angle of orientation and is calculated using squared gradient averaging method in [9], centered at $(x, y)$ with a certain radius as the region of interest (ROI). Then the ROI is divided into $N$ homocentric 

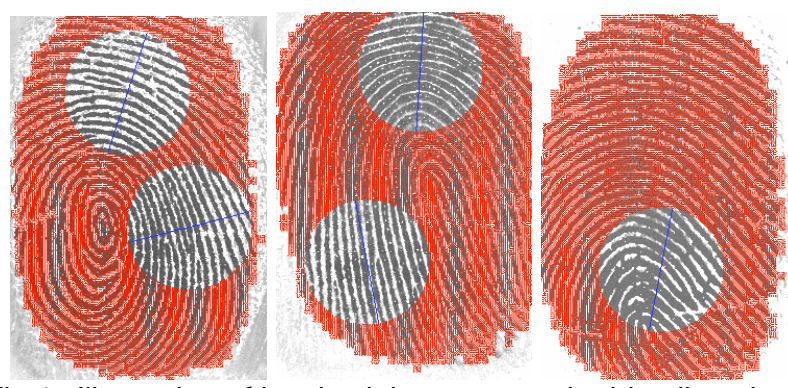

Fig 1. Illustration of local axial symmetry: the blue lines in the circles represent their optimized symmetry axes.

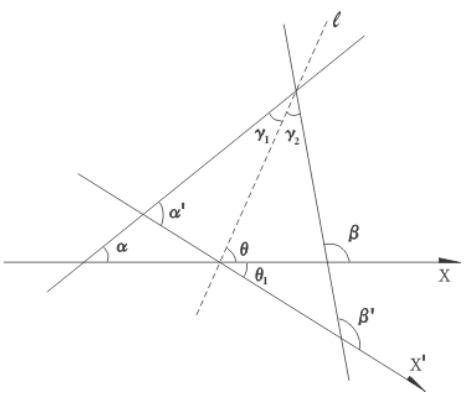

Fig 2 Demonstration of the axial symmetry's condition: $x$ is the $x$-axis of the image, $x^{\prime}$ is the supposed rotated $x$-axis, $l$ is the candidate symmetry axis, $\theta$ is the direction angle of $l$, $\alpha, \alpha^{\prime}, \beta$ and $\beta^{\prime}$ are the average orientations of the sector pair (half of AOS) in the two coordinate systems.

sectors with a series of equally distributed radii which are also the set of candidate symmetry axes of the region.

The average orientation of every sector, namely AOS, is calculated according to (1) and (2), where $S_{i}$ is the $i$-th sector of the ROI and $\left|S_{i}\right|$ is the number of pixels in $S_{i}$.

$$
\begin{gathered}
{\left[\begin{array}{l}
\operatorname{AOS}_{x}\left(S_{i}\right) \\
\operatorname{AOS}_{y}\left(S_{i}\right)
\end{array}\right]=\left[\begin{array}{l}
\frac{1}{\left|S_{i}\right|} \sum_{(x, y) \in S_{i}} P O F_{x}(x, y) \\
\frac{1}{\left|S_{i}\right|} \sum_{(x, y) \in S_{i}} \operatorname{POF}_{y}(x, y)
\end{array}\right]} \\
\operatorname{AOS}\left(S_{i}\right)=\arctan 2\left(\operatorname{AOS}_{y}\left(S_{i}\right), \operatorname{AOS}_{x}\left(S_{i}\right)\right)
\end{gathered}
$$

Consider the $i$-th candidate axis which corresponding direction is $\frac{i * 2 * P I}{N}$. The counterpart sector of $S_{i+j}$ is $S_{i-j-1}$. As demonstrated in Fig 2, if the sector pair which orientations are $\alpha$ and $\beta$ take a line of direction $\theta$ as their symmetry axis, (3) must be fulfilled. Thus we can use $\cos (2 \alpha+2 \beta-4 \theta)$ to weigh the local axial symmetry: the larger this value is, the more axial symmetry with regard to $l$ can be expected.

$$
\begin{aligned}
& \gamma_{1}=\gamma_{2} \Leftrightarrow \theta-\alpha=\beta-\theta \\
& \Leftrightarrow \alpha+\beta-2 \theta=0 \Leftrightarrow 2 \alpha+2 \beta-4 \theta=0
\end{aligned}
$$

In fact $\operatorname{AOS}\left(S_{i}\right)$ is twice of the sector $S_{i}$ 's orientation, so symmetry value with respect to $i$-th candidate axis and local axial symmetry of point $(x, y)$ are defined as (4) and (5) respectively. The corresponding orientation of $\hat{i}$ which maximizes (4) gives the symmetry axis of this region.

$$
\begin{aligned}
& \operatorname{Sym}(i)=\frac{2}{N} \sum_{j=0}^{N / 2-1} \cos \left[\operatorname{AOS}\left(S_{i+j}\right)+\operatorname{AOS}\left(S_{i-j-1}\right)-\frac{4 * i * 2 * P I}{N}\right] \\
& \operatorname{LAS}(x, y)=\max \{\operatorname{Sym}(i) \mid i \in[0,1, . . N / 2)\}
\end{aligned}
$$

Suppose the $\mathrm{x}$-axis rotates to $x^{\prime}$. As proved in (6), $\cos (2 \alpha+2 \beta-4 \theta)$ is invariant through the rotation. That is to say, the LAS is rotation-independent. Obviously it is translation-independent too according to its definition.

$$
\begin{aligned}
& \because \alpha^{\prime}=\alpha+\theta_{1}, \beta^{\prime}=\beta+\theta_{1}, \theta^{\prime}=\theta+\theta_{1} \\
& \therefore 2 \alpha^{\prime}+2 \beta^{\prime}-4 \theta^{\prime}=2 \alpha+2 \beta-4 \theta
\end{aligned}
$$

The consideration of choosing circle region as ROI is the spatial isotropy and of choosing average orientation of a sector as the feature element is to facilitate the selection of the symmetry axis. The radius describes the range of ROI which implies the degree of LAS's locality.

\section{REFERENCE POINT DETECTION AND INDEXING}

Now we are about to apply our new feature to detect reference points of fingerprint images. First the image is segmented and the POF is computed. As mentioned above, POF is very noise-sensitive. So a smoothing must be performed first to restrain it. Here we choose Gaussian filter to gain our ends. For FVC2000 DB2 the $\sigma$ of filter is set to 20. Experimental results show that this strength of smoothing can delete most noise and keep enough information for efficient LAS calculation.

To reduce the computational complexity, the foreground is partitioned into several square blocks of equal size. The choice of the size is a tradeoff between speed and localization accuracy. Here we set it to 4. Note that POF after Gaussian smoothing is continuous, so in each small block LAS value and direction is approximately equal. Upon that the LAS of the block's center is calculated as LAS of the block. After this process, the fingerprint image is converted to a discrete matrix of LAS that we entitle it "LAS field".

As can be easily seen in Fig 3, in LAS fields of different qualities and types of fingerprint images including the plain arch, there are two regions taking on lower LAS value on the two opposite sides of the reference point. Many experiments have shown this character is an inherent nature of fingerprint LAS fields except that the reference point or at least one of these two regions is out of the foreground. 


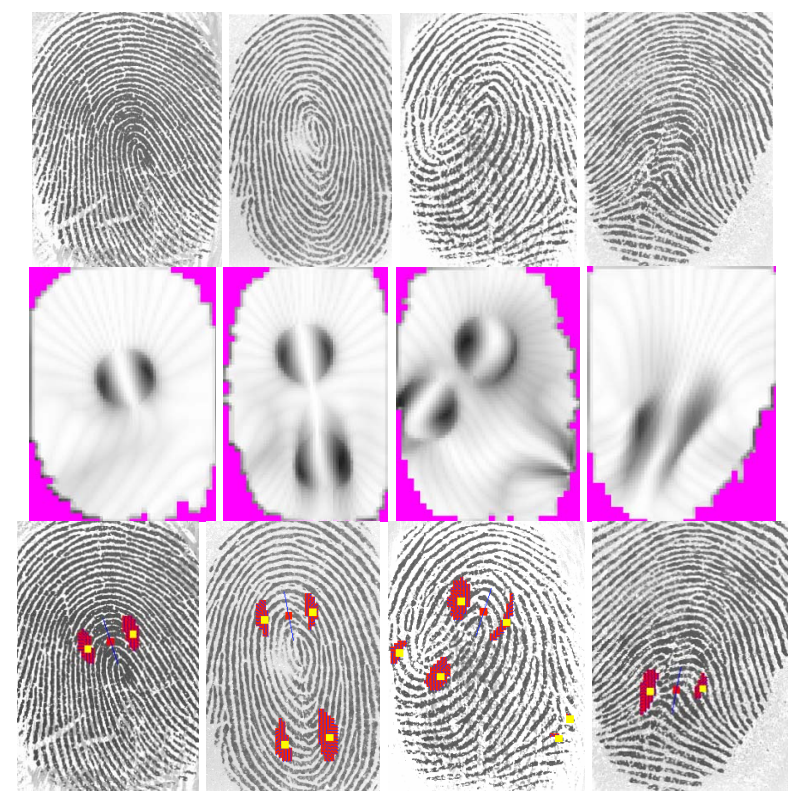

Fig 3 Illustration of reference point detection process: images in the first row are the original fingerprints; images in the middle row are corresponding LAS field where the grayscales represent the corresponding blocks' LAS; images in last row are binarized images according the LAS with detected reference points with their direction indicated by line segments where the yellow squares represent the center of corresponding foreground region.

These two regions are so reliable that we can obtain them by a simple binarization through a constant threshold. Then we take the upmost two low LAS regions' centers of gravity as landmarks and take their midpoint as the reference point. The direction of the reference point is estimated as the direction of symmetry axis of the corresponding block.

Due to smoothing effect, the reference point localization result may be translated from its conventional positions, which often further brings varying in the following direction estimation step. Fortunately we find that these offsets are systematic errors as to the same finger and similar smoothing intensity. That is to say, for different images of the same finger, their reference points always "move" along the same track to the same destinations in the registered coordinate systems during the smoothing process. The direction estimation is also dependable for it is the same region's symmetry axis if the corresponding fingers are identical. Experimental results have proven that this type of systematic errors scarcely deteriorate the indexing efficiency.

It is well-known that the OF contains abundant information to discriminate fingerprints. It can even be utilized to perform fingerprint matching [10]. In our view, if the reliable transformation of registration is achieved and the proper scale is chosen, BOF can be utilized to measure the fingerprint similarity. Upon that our indexing algorithm is presented.

To restrain the noise effect, the BOF is first smoothed by Gaussian filter. Then it is translated to center at the detected reference point and rotated to parallel to the direction of it. Next, the registered BOF data ( $\sin$ and cos values of the doubled orientation angle) in constant region are extracted to constitute the feature vectors for indexing. Considering the dimension of feature vectors and their discriminability synthetically, the size of block is chosen to $16 \times 16$. Note that to the block out of the foreground region, its position is recorded and its corresponding date are set to a nonsensical value. The indexing is performed based on the Euclidian distances between the fingerprints in the testing set which labels are to be determined and all labeled fingerprints in the training set.

\section{EXPERIMENTAL RESULTS}

We have run our experiments on the second database of the FVC2000 [11]. In this database, 100 untrained individuals are enrolled, each with eight prints of the same finger. Due to the comparatively larger number of prints with same label (sequence number of finger), we introduce a special experimental scheme. To each finger, a certain "Number of prints with Same Label" (NSL for short) are chosen to construct the training set. The NSL in our experiments is not constant like other algorithms do and its value is set to be 1 to 6 .

First the proposed algorithm is applied to all the 800 fingerprints in the database and 12 images of them are refused due to the absence of the reference points or at least one region with the lower LAS value. And then their registered OFs -- the feature vectors to measure the similarity to other prints are obtained. To a certain NSL, the whole database is partitioned into the training set (the first NSL prints of all fingers) and the testing set (the other samples). The prints in the testing set are processed one by one as follows:

The Euclid distances between the feature vector of the current testing print and all prints in the training set are calculated. Note that the distance is calculated on the elements which values in both feature vectors are valid, and normalized considering the size of the intersection. Then all prints in the training set are sorted ascending to a prints sequence by above distance with respect to the current testing print. Further all fingers are sorted to a finger sequence according to their first occurrence in abovementioned sequence. The position of the testing print's corresponding finger in the sequence (namely indexing rank) describes the indexing algorithm's effect on this testing print and determines the number of matching operation in a fingerprint based person authentication systems.

The Correct Index Power [2] (CIP) curves of different NSL are shown in Fig 4. As can be easily seen, the larger NSL is, the better results are obtained. It indicates that the sufficient training can consistently improve our algorithm's 


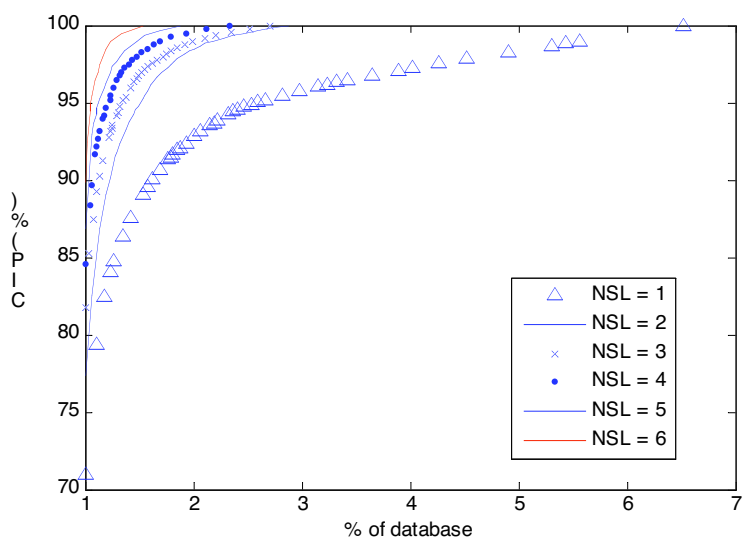

Fig 4 CIP curve of difference NSL values

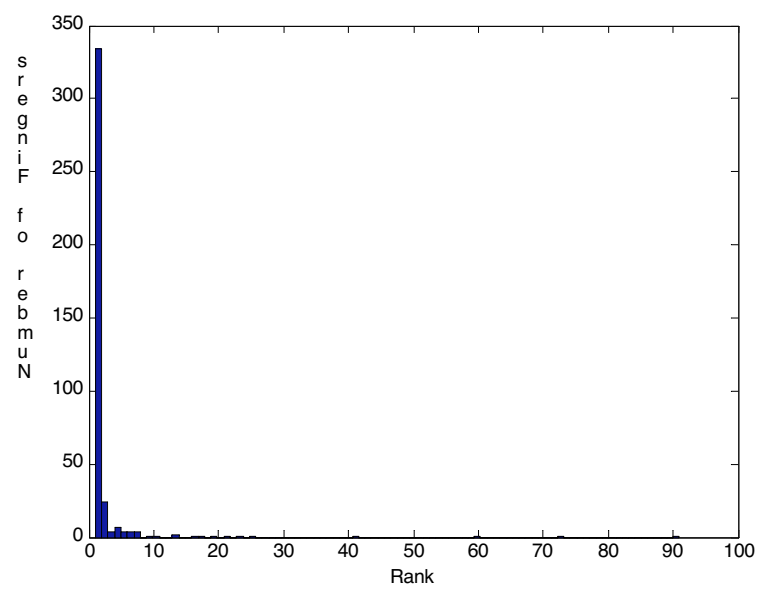

Fig 5 Histogram of the rank distribution of NSL 4

performance.

We also list in detail the experimental results in the condition of NSL=4 (namely the training set and the testing set are of equal size) as a typical view of our algorithms. Fig 5 is the histogram of the indexing rank value of $N S L=4$.

In this condition, 395 prints from the total 400 testing prints are registered using our LAS algorithm. The refusal rate is $1.25 \%$. Within the 395 prints, $334(84.56 \%)$ prints get rank 1 . The rate of prints which rank are larger than 7 is only $14(3.54 \%)$ of the whole testing set. The mean rank is 2.34 which means by just searching the $2.34 \%$ of the database the true finger will be found in an average sense.

\section{CONCLUSION}

In this paper we propose an efficient fingerprint indexing algorithm using the registered OFs as feature vectors to measure the similarity of fingerprints. The registration is performed based on a novel feature of fingerprint namely local axial symmetry.
Experimental results show our indexing can remarkably reduce the workload of the fingerprint authentication system. The size of training set is also analyzed through experiments of different NSL value. It is testified that the efficiency can be consistently promoted when the training set grows larger.

\section{ACKNOWLEDGMENTS}

This work is supported by research funds from NSFC No. 60572043 , the NKBRPC No.2004CB318005 and FANEDD No. 200038.

\section{REFERENCES}

[1] K. Choi, D. Lee, S. Lee, and J. Kim, "An Improved Fingerprint Indexing Algorithm Based on the Triplet Approach", pp. 584-591, AVBPA 2003.

[2] X. Tan, B. Bhanu, and Y. Lin, "Fingerprint Identification: Classification vs. Indexing", pp. 151-156, AVSS 2003.

[3] B Bhanu, and X Tan, "Fingerprint indexing based on novel features of minutiae triplets", Pattern Analysis and Machine Intelligence, IEEE Transactions on, Vol. 25, Issue 5, pp. 616- 622, 2003.

[4] A.K. Jain, S. Prabhakar, L. Hong, and S. Pankanti, "FingerCode: a filterbank for fingerprint representation and matching", Computer Vision and Pattern Recognition IEEE Computer Society Conference, Vol. 2, pp. 187-193, 1999.

[5] T. Liu, G. Zhu, C. Zhang and P. Hao, "Fingerprint Indexing Based on Singular Point Correlation", Image Processing, 2005. ICIP 2005. IEEE International Conference on Vol. 3, pp. 293-296, 2005.

[6] D. Maltoni, D. Maio, A.K. Jain, S. Prabhakar, "Handbook of Fingerprint Recognition", Springer-Verlag, New York 2003.

[7] L. Sha and X. Tang, "Combining exclusive and continuous fingerprint classification", IEEE International Conference on Image Processing, pp. 1245-1248, 2004.

[8] M. Kawagoe, A. Tojo, "Fingerprint Pattern Classification", Pattern Recognition, Vol. 17, no. 3, pp. 295-303, 1984

[9] A. Bazen, S. Gerez, "Systematic Methods for the Computation of the Directional Fields and Singular Points of Fingerprints", IEEE Transaction on Pattern Analysis and Machine Intelligence, Vol.24, no.7, pp. 905-919, 2002.

[10] N. Yager, A. Amin. "Evaluation of fingerprint orientation field registration algorithms", Proceedings of the 17th International Conference on Pattern Recognition, Vol. 4, pp.641-644, 2004.

[11] D. Maio, D. Maltoni, R. Cappelli, J.L. Wayman, and A.K. Jain, "FVC2000: Fingerprint Verification Competition," Biolab internal report, Univ. of Bologna, Italy, 2000. 